\title{
Nontrivial Solutions for a Higher Order Nonlinear Fractional Boundary Value Problem Involving Riemann-Liouville Fractional Derivatives
}

\author{
Keyu Zhang $\left(\mathbb{D},{ }^{1}\right.$ Donal O'Regan, ${ }^{2}$ Jiafa Xu $\left(\mathbb{D},{ }^{3}\right.$ and Zhengqing Fu $\mathbb{D}^{4}$ \\ ${ }^{1}$ School of Mathematics, Qilu Normal University, Jinan 250013, China \\ ${ }^{2}$ School of Mathematics, Statistics and Applied Mathematics, National University of Ireland, Galway, Ireland \\ ${ }^{3}$ School of Mathematical Sciences, Chongqing Normal University, Chongqing 401331, China \\ ${ }^{4}$ College of Mathematics and System Sciences, Shandong University of Science and Technology, Qingdao 266590, China \\ Correspondence should be addressed to Keyu Zhang; keyu_292@163.com
}

Received 18 March 2019; Revised 11 April 2019; Accepted 14 April 2019; Published 19 June 2019

Academic Editor: Raúl E. Curto

Copyright (C) 2019 Keyu Zhang et al. This is an open access article distributed under the Creative Commons Attribution License, which permits unrestricted use, distribution, and reproduction in any medium, provided the original work is properly cited.

In this paper using topological degree we study the existence of nontrivial solutions for a higher order nonlinear fractional boundary value problem involving Riemann-Liouville fractional derivatives. Here, the nonlinearity can be sign-changing and can also depend on the derivatives of unknown functions.

\section{Introduction}

In this paper, we investigate the existence of nontrivial solutions for the higher order nonlinear fractional boundary value problem involving Riemann-Liouville fractional derivatives:

$$
\begin{aligned}
& \begin{array}{l}
D_{0+}^{\alpha} u(t) \\
=-f\left(t, u(t), D_{0+}^{\beta_{1}} u(t), D_{0+}^{\beta_{2}} u(t), \ldots, D_{0+}^{\beta_{n-1}} u(t)\right), \\
0<t<1,
\end{array} \\
& u(0)=u^{\prime}(0)=\cdots=u^{(n-2)}(0)=D_{0+}^{\beta} u(1)=0,
\end{aligned}
$$

where $n-1<\alpha \leq n, i-1<\beta_{i} \leq i, \alpha-\beta_{n-1}>\alpha-$ $\beta>1, D_{0+}^{\alpha}, D_{0+}^{\beta}, D_{0+}^{\beta_{i}}$ are the Riemann-Liouville fractional derivatives, $i=1,2, \ldots, n-1$, and the function $f:[0,1] \times$ $\mathbb{R}^{n} \longrightarrow \mathbb{R}$ is continuous; here $\mathbb{R}:=(-\infty,+\infty)$.

There are a large number of papers in the literature studying fractional differential equations because of applications in various disciplines of science and engineering such as mechanics, electricity, chemistry, and control theory; we refer the reader to [1-35] and the references therein. In [1], using fixed point index theory in cones, the authors studied the existence and multiplicity of positive solutions for the nonlocal fractional differential equation boundary value problem

$$
\begin{aligned}
& D_{0+}^{\alpha} u(t)+q(t) f(t, u(t))=0, \\
& 0<t<1, n-1<\alpha \leq n, \\
& u(0)=u^{\prime}(0)=\cdots=u^{(n-2)}(0)=0, \\
& u(1)=\int_{0}^{1} u(s) d A(s),
\end{aligned}
$$

where $f(t, u)$ is a nonnegative continuous function on $[0,1] \times$ $\mathbb{R}^{+}$and satisfies the conditions:

(H1) Wang sublinear growth case: $\liminf _{u \rightarrow 0^{+}}(f(t, u) /$ $u)>\lambda_{1}, \lim \sup _{u \rightarrow+\infty}(f(t, u) / u)<\lambda_{1}$, uniformly on $t \in$ $[0,1]$,

$(\mathrm{H} 2)_{\text {Wang }}$ superlinear growth case: $\liminf _{u \rightarrow+\infty}(f(t$, $u) / u)>\lambda_{1}, \lim \sup _{u \rightarrow 0^{+}}(f(t, u) / u)<\lambda_{1}$, uniformly on $t \in[0,1]$, 
where $\lambda_{1}$ is the first eigenvalue for the problem:

$$
\begin{aligned}
D_{0+}^{\alpha} u(t)+\lambda q(t) u(t) & =0, \quad 0<t<1, \\
u(0) & =u^{\prime}(0)=\cdots=u^{(n-2)}(0)=0 \\
u(1) & =\int_{0}^{1} u(s) d A(s) .
\end{aligned}
$$

Conditions similar to $(\mathrm{H} 1)_{\text {Wang }}$ and $(\mathrm{H} 2)_{\text {Wang }}$ can be found in [2-9]. In [2], the authors studied the existence of positive solutions for the nonlinear fractional differential equation:

$$
\begin{aligned}
-D_{0+}^{\alpha} y(t) & =f\left(t, y(t), y^{\prime}(t), \ldots, y^{(n-2)}(t)\right), \\
0<t<1, & \\
y(0) & =y^{\prime}(0)=\cdots=y^{(n-2)}(0)=0, \\
y^{(n-2)}(1) & =\lambda\left[y^{(n-2)}\right],
\end{aligned}
$$

where $\lambda\left[y^{(n-2)}\right]=\int_{0}^{1} y^{(n-2)}(t) d A(t)$ is a linear functional on $C[0,1]$ given by a Stieltjes integral. The nonlinearity $f\left(t, z_{1}, z_{2}, \ldots, z_{n-1}\right)$ satisfies the conditions:

$$
\begin{gathered}
\liminf _{z_{1} \longrightarrow 0, \ldots, z_{n-1} \rightarrow 0} \frac{f\left(t, z_{1}, z_{2}, \ldots, z_{n-1}\right)}{z_{1}+\cdots+z_{n-1}}>\lambda_{1}, \\
\limsup _{z_{1}+\cdots+z_{n-1} \longrightarrow+\infty, z_{n-1} \longrightarrow+\infty} \frac{f\left(t, z_{1}, z_{2}, \ldots, z_{n-1}\right)}{z_{n-1}}<\lambda_{1}, \\
\text { uniformly on } t \in[0,1],
\end{gathered}
$$

and

$$
\begin{aligned}
& \liminf _{z_{1} \rightarrow 0, \ldots, z_{n-1} \rightarrow 0} \frac{f\left(t, z_{1}, z_{2}, \ldots, z_{n-1}\right)}{z_{n-1}}<\lambda_{1}, \\
& \limsup _{z_{1}+\cdots+z_{n-1} \longrightarrow+\infty} \frac{f\left(t, z_{1}, z_{2}, \ldots, z_{n-1}\right)}{z_{1}+\cdots+z_{n-1}}>\lambda_{1}, \\
& \text { uniformly on } t \in[0,1] .
\end{aligned}
$$

In [10-14], the authors used Lipschitz conditions to study the existence and uniqueness of solutions for various fractional differential equations; for example, in [10], the author used the $u_{0}$-positive operator method to obtain the unique solution for the fractional boundary value problem:

$$
\begin{aligned}
D_{t}^{p} x(t)+p(t) f(t, x(t))+q(t) & =0, \quad t \in(0,1), \\
x(0) & =x^{\prime}(0)=0, \\
x(1) & =0,
\end{aligned}
$$

where $f$ is a Lipschitz continuous function, with the Lipschitz constant associated with the first eigenvalue for the relevant operator. In [15], the authors used the mixed monotone method to obtain a unique positive solution for the fractional boundary value problem:

$$
\begin{array}{r}
-D_{0+}^{\alpha} y(t)=q(t) f\left(t, y(t), y^{\prime}(t), \ldots, y^{(n-2)}(t)\right), \\
0<t<1, \\
y(0)=y^{\prime}(0)=\cdots=y^{(n-2)}(0)=y^{(n-2)}(1)=0,
\end{array}
$$

where the nonlinearity $f=g+h$ and $g, h$ have different monotone properties. In $[16,17]$, the authors extended the methods in [15] to fractional p-Laplacian equations and established some appropriate iterative sequences which converge to their solutions.

There are also some papers in the literature devoted to sign-changing nonlinearities; we refer the reader to [7-14, 18, $19,22,23,26-33,35]$. For example, in [18] using coincidence degree theory, the author investigated the existence of nontrivial solutions for the coupled system of nonlinear fractional differential equations:

$$
\begin{aligned}
D_{0+}^{\alpha} u(t) & =f\left(t, v(t), D_{0+}^{\beta-2} v(t), D_{0+}^{\beta-1} v(t)\right), \\
D_{0+}^{\beta} v(t) & =g\left(t, u(t), D_{0+}^{\alpha-2} u(t), D_{0+}^{\alpha-1} u(t)\right), \\
u(0) & =0, \\
D_{0+}^{\alpha-1} u(0) & =D_{0+}^{\alpha-1} u(\eta), \\
u(1) & =\sum_{i=1}^{m_{1}} \alpha_{i} u\left(\eta_{i}\right), \\
v(0) & =0, \\
D_{0+}^{\beta-1} v(0) & =D_{0+}^{\beta-1} v(\xi), \\
v(1) & =\sum_{i=1}^{m_{2}} \beta_{i} v\left(\xi_{i}\right),
\end{aligned}
$$

where $f, g:[0,1] \times \mathbb{R}^{3} \longrightarrow \mathbb{R}$ satisfy Carathéodory conditions.

Motivated by the aforementioned papers and some integer order equations [36-39], in this paper we use topological degree to study the existence of nontrivial solutions for the higher order nonlinear fractional boundary value problem (1). Our nonlinearity $f$ can be sign-changing and can also depend on the derivatives of unknown functions. Moreover, our conditions improve the conditions in (5) and (6); see (H4)-(H5) in Section 3.

\section{Preliminaries}

We present some basic material involving Riemann-Liouville fractional derivatives; for details we refer the reader to the books [40-42]. 
Definition 1 (see [40-42]). The Riemann-Liouville fractional derivative of order $\alpha>0$ of a continuous function $f$ : $(0,+\infty) \longrightarrow(-\infty,+\infty)$ is given by

$$
D_{0+}^{\alpha} f(t)=\frac{1}{\Gamma(n-\alpha)}\left(\frac{\mathrm{d}}{\mathrm{d} t}\right)^{n} \int_{0}^{t}(t-s)^{n-\alpha-1} f(s) d s,
$$

where $n=[\alpha]+1,[\alpha]$ denotes the integer part of number $\alpha$, provided that the right side is pointwise defined on $(0,+\infty)$.

Definition 2 (see [40-42]). The Riemann-Liouville fractional integral of order $\alpha>0$ of a function $f:(0,+\infty) \longrightarrow$ $(-\infty,+\infty)$ is given by

$$
I_{0+}^{\alpha} f(t)=\frac{1}{\Gamma(\alpha)} \int_{0}^{t}(t-s)^{\alpha-1} f(s) d s,
$$

provided that the right side is pointwise defined on $(0,+\infty)$.

Lemma 3 (see [3, Lemma 2.2]). Let $\alpha>0$, then for $u, D_{0+}^{\alpha} u \in$ $C(0,1) \cap L(0,1)$, we have

$$
\begin{array}{r}
I_{0+}^{\alpha} D_{0+}^{\alpha} u(t)=u(t)+c_{1} t^{\alpha-1}+c_{2} t^{\alpha-2}+\ldots+c_{N} t^{\alpha-N}, \\
\text { for some } c_{i} \in \mathbb{R}, i=1,2, \ldots, N,
\end{array}
$$

where $N$ is the smallest integer greater than or equal to $\alpha$.

In what follows, we present Green's function associated with problem (1). The following lemma is from [3]; for completeness we provide the proof.

Lemma 4 (see [3, Lemma 2.3]). Let $v(t)=D_{0+}^{\beta_{n-1}} u(t)$. Then (1) can be transformed into the following nonlinear integrodifferential equation:

$$
\begin{aligned}
& D_{0+}^{\alpha-\beta_{n-1}} v(t)=-f\left(t, I_{0+}^{\beta_{n-1}} v(t), I_{0+}^{\beta_{n-1}-\beta_{1}} v(t), \ldots,\right. \\
& \left.\quad I_{0+}^{\beta_{n-1}-\beta_{n-2}} v(t), v(t)\right), \quad 0<t<1, \\
& I_{0+}^{\beta_{n-1}-n+2} v(0)=D_{0+}^{\beta-\beta_{n-1}} v(1)=0 .
\end{aligned}
$$

Consequently, we obtain that (13) is equivalent to the following Hammerstein type integral equation:

$$
\begin{gathered}
v(t)=\int_{0}^{1} K_{1}(t, s) f\left(s, I_{0+}^{\beta_{n-1}} v(s), I_{0+}^{\beta_{n-1}-\beta_{1}} v(s), \ldots,\right. \\
\left.I_{0+}^{\beta_{n-1}-\beta_{n-2}} v(s), v(s)\right) d s
\end{gathered}
$$

where

$$
\begin{aligned}
& K_{1}(t, s)=\frac{1}{\Gamma\left(\alpha-\beta_{n-1}\right)} \\
& \quad \cdot \begin{cases}t^{\alpha-\beta_{n-1}-1}(1-s)^{\alpha-\beta-1}-(t-s)^{\alpha-\beta_{n-1}-1}, & 0 \leq s \leq t \leq 1, \\
t^{\alpha-\beta_{n-1}-1}(1-s)^{\alpha-\beta-1}, & 0 \leq t \leq s \leq 1 .\end{cases}
\end{aligned}
$$

Proof. Let $v(t)=D_{0+}^{\beta_{n-1}} u(t)$. Then from [4, equations (2.11)], we have

$$
\begin{gathered}
D_{0+}^{\alpha} u(t)=D_{0+}^{\alpha-\beta_{n-1}} v(t), \\
D_{0+}^{\beta_{1}} u(t)=D_{0+}^{\beta_{n-1}-\beta_{1}} v(t), \\
D_{0+}^{\beta_{2}} u(t)=D_{0+}^{\beta_{n-1}-\beta_{2}} v(t), \\
\cdots, \\
D_{0+}^{\beta_{n-2}} u(t)=D_{0+}^{\beta_{n-1}-\beta_{n-2}} v(t), \\
D_{0+}^{\beta_{n-1}} u(t)=v(t) .
\end{gathered}
$$

Also we obtain $u^{(n-2)}(t)=I_{0+}^{\beta_{n-1}-n+2} v(t)$, and $D_{0+}^{\beta} u(t)=$ $D_{0+}^{\beta-\beta_{n-1}} v(t)$. Hence, by $u(t)=I_{0+}^{\beta_{n-1}} v(t)$, we have (13). Consequently, Lemma 3 implies that (13) can be reduced to an equivalent integral equation:

$$
\begin{gathered}
v(t)=-I_{0+}^{\alpha-\beta_{n-1}} f\left(t, I_{0+}^{\beta_{n-1}} v(t), I_{0+}^{\beta_{n-1}-\beta_{1}} v(t), \ldots,\right. \\
\left.I_{0+}^{\beta_{n-1}-\beta_{n-2}} v(t), v(t)\right)+C_{1} t^{\alpha-\beta_{n-1}-1}+C_{2} t^{\alpha-\beta_{n-1}-2},
\end{gathered}
$$

for some $C_{i} \in \mathbb{R}, i=1,2$. From the condition $I_{0+}^{\beta_{n-1}-n+2} v(0)=$ 0 , we have $C_{2}=0$. Then we obtain

$$
\begin{aligned}
D_{0+}^{\beta-\beta_{n-1}} v(t)= & -\frac{1}{\Gamma(\alpha-\beta)} \int_{0}^{t}(t-s)^{\alpha-\beta-1} \tilde{f}(s) d s \\
& +C_{1} \frac{\Gamma\left(\alpha-\beta_{n-1}\right)}{\Gamma(\alpha-\beta)} t^{\alpha-\beta-1},
\end{aligned}
$$

where we use $\tilde{f}(t)$ to replace $f(t, \cdot, \cdots, \cdot)$. Then $D_{0+}^{\beta-\beta_{n-1}} v(1)=0$ solves the above equation, and we obtain

$$
C_{1}=\frac{1}{\Gamma\left(\alpha-\beta_{n-1}\right)} \int_{0}^{1}(1-s)^{\alpha-\beta-1} \tilde{f}(s) d s .
$$

Therefore, we have

$$
\begin{aligned}
v(t) & \\
= & -I_{0+}^{\alpha-\beta_{n-1}} \tilde{f}(t) \\
& +\frac{1}{\Gamma\left(\alpha-\beta_{n-1}\right)} \int_{0}^{1} t^{\alpha-\beta_{n-1}-1}(1-s)^{\alpha-\beta-1} \tilde{f}(s) d s \\
= & -\frac{1}{\Gamma\left(\alpha-\beta_{n-1}\right)} \int_{0}^{t}(t-s)^{\alpha-\beta_{n-1}-1} \tilde{f}(s) d s \\
& +\frac{1}{\Gamma\left(\alpha-\beta_{n-1}\right)} \int_{0}^{1} t^{\alpha-\beta_{n-1}-1}(1-s)^{\alpha-\beta-1} \tilde{f}(s) d s \\
= & \int_{0}^{1} K_{1}(t, s) \tilde{f}(s) d s .
\end{aligned}
$$

This completes the proof. 
Lemma 5 (see [3, Lemma 2.4]). The function $K_{1}$ has the following properties:

(i) $K_{1}:[0,1] \times[0,1] \longrightarrow \mathbb{R}^{+}$is continuous,

(ii) for all $(t, s) \in[0,1] \times[0,1], t^{\alpha-\beta_{n-1}-1} K_{1}(1, s) \leq$ $K_{1}(t, s) \leq K_{1}(1, s)$, where $K_{1}(1, s)=\left((1-s)^{\alpha-\beta-1}-(1-\right.$ $\left.s)^{\alpha-\beta_{n-1}-1}\right) / \Gamma\left(\alpha-\beta_{n-1}\right)$.

Let $E:=C[0,1],\|v\|:=\max _{t \in[0,1]}|v(t)|, P:=\{v \in E:$ $v(t) \geqslant 0, \forall t \in[0,1]\}, P_{0}=\left\{v \in P: v(t) \geq t^{\alpha-\beta_{n-1}-1}\|v\|, \forall t \in\right.$ $[0,1]\}$. Then $(E,\|\cdot\|)$ is a real Banach space, and $P, P_{0}$ are cones on E. From Lemma 4, we can define an operator $A: E \longrightarrow E$ as follows:

$$
\begin{gathered}
(A v)(t)=\int_{0}^{1} K_{1}(t, s) f\left(s, I_{0+}^{\beta_{n-1}} v(s), I_{0+}^{\beta_{n-1}-\beta_{1}} v(s), \ldots,\right. \\
\left.I_{0+}^{\beta_{n-1}-\beta_{n-2}} v(s), v(s)\right) d s, \quad \text { for } v \in E
\end{gathered}
$$

where $K_{1}$ is denoted by (15). Note that our functions $K_{1}, f$ are continuous, so the operator $A$ is a completely continuous operator. Moreover if there is $a \bar{v} \in E$ as a fixed point of $A$, i.e., (13) has s solution $\bar{v}$, then from $\bar{v}(t)=D_{0+}^{\beta_{n-1}} \bar{u}(t)$, we have that $\bar{u}$ is a solution for (1). Therefore we study the existence of fixed points of the operator A. For n nonnegative real numbers $\mu_{1}, \mu_{2}, \ldots, \mu_{n}$ which are not all zero, let

$$
\left(L_{\mu_{1}, \mu_{2}, \ldots, \mu_{n}} v\right)(t)=\int_{0}^{1} K_{\mu_{1}, \mu_{2}, \ldots, \mu_{n}}(t, s) v(s) d s
$$

\section{for $v \in E$,}

where $K_{\mu_{1}, \mu_{2}, \ldots, \mu_{n}}(t, s)=\mu_{n} K_{n}(t, s)+\mu_{n-1} K_{n-1}(t, s)+\cdots+$ $\mu_{1} K_{1}(t, s)$ for $(t, s) \in[0,1] \times[0,1]$, and $K_{i}(i=2, \ldots, n)$ are

$$
\begin{aligned}
& {\left[\begin{array}{c}
K_{2}(t, s) \\
K_{3}(t, s) \\
\vdots \\
K_{n-1}(t, s) \\
K_{n}(t, s)
\end{array}\right]} \\
& =\left[\begin{array}{c}
\frac{1}{\Gamma\left(\beta_{n-1}-\beta_{n-2}\right)} \int_{0}^{\tau} K_{1}(t, \tau)(\tau-s)^{\beta_{n-1}-\beta_{n-2}-1} d \tau \\
\frac{1}{\Gamma\left(\beta_{n-1}-\beta_{n-3}\right)} \int_{0}^{\tau} K_{1}(t, \tau)(\tau-s)^{\beta_{n-1}-\beta_{n-3}-1} d \tau \\
\frac{1}{\Gamma\left(\beta_{n-1}-\beta_{1}\right)} \int_{0}^{\tau} K_{1}(t, \tau)(\tau-s)^{\beta_{n-1}-\beta_{1}-1} d \tau \\
\frac{1}{\Gamma\left(\beta_{n-1}\right)} \int_{0}^{\tau} K_{1}(t, \tau)(\tau-s)^{\beta_{n-1}-1} d \tau
\end{array}\right],
\end{aligned}
$$$$
\text { for } t, s \in[0,1] \text {. }
$$

Let $r\left(L_{\mu_{1}, \mu_{2}, \ldots, \mu_{n}}\right)$ denote the spectral radius of $L_{\mu_{1}, \mu_{2}, \ldots, \mu_{n}}$, and from Gelfand's theorem we can prove $r\left(L_{\mu_{1}, \mu_{2}, \ldots, \mu_{n}}\right)>0$ (the proof is standard; see [6, Lemma 5]).
Define an operator $A_{1}: E \longrightarrow E$ as follows:

$$
\left(A_{1} z\right)(t)=\int_{0}^{1} K_{1}(t, s) z(s) d s, \quad \text { for } z \in E \text {. }
$$

Then from Lemma 5(ii), we have

$$
A_{1}(P) \subset P_{0}
$$

Lemma 6 (see [43, Theorem 19.3]). Let $P$ be a reproducing cone in a real Banach space $E$ and let $L: E \longrightarrow E$ be a compact linear operator with $L(P) \subset P$. Let $r(L)$ be the spectral radius of $L$. If $r(L)>0$, then there exists $\varphi \in P \backslash\{0\}$ such that $L \varphi=r(L) \varphi$.

Therefore, from Lemma 6 there exists $\varphi_{\mu_{1}, \mu_{2}, \ldots, \mu_{n}} \in P \backslash\{0\}$ such that

$$
L_{\mu_{1}, \mu_{2}, \ldots, \mu_{n}} \varphi_{\mu_{1}, \mu_{2}, \ldots, \mu_{n}}=r\left(L_{\mu_{1}, \mu_{2}, \ldots, \mu_{n}}\right) \varphi_{\mu_{1}, \mu_{2}, \ldots, \mu_{n}} .
$$

Hence, we obtain

$$
\begin{gathered}
\varphi_{\mu_{1}, \mu_{2}, \ldots, \mu_{n}}(t)=\frac{1}{r\left(L_{\mu_{1}, \mu_{2}, \ldots, \mu_{n}}\right)}\left(L_{\mu_{1}, \mu_{2}, \ldots, \mu_{n}} \varphi_{\mu_{1}, \mu_{2}, \ldots, \mu_{n}}\right)(t) \\
=\frac{1}{r\left(L_{\mu_{1}, \mu_{2}, \ldots, \mu_{n}}\right)} \int_{0}^{1} K_{\mu_{1}, \mu_{2}, \ldots, \mu_{n}}(t, s) \varphi_{\mu_{1}, \mu_{2}, \ldots, \mu_{n}}(s) d s \\
=\frac{1}{r\left(L_{\mu_{1}, \mu_{2}, \ldots, \mu_{n}}\right)} \int_{0}^{1}\left[\mu_{n} K_{n}(t, s)+\mu_{n-1} K_{n-1}(t, s)\right. \\
\left.+\cdots+\mu_{1} K_{1}(t, s)\right] \varphi_{\mu_{1}, \mu_{2}, \ldots, \mu_{n}}(s) d s, \quad t \in[0,1] .
\end{gathered}
$$

Using Lemma 5(ii) and the definitions of $K_{i}(i=1,2, \ldots, n)$, we have

$$
\begin{aligned}
& \left\|\varphi_{\mu_{1}, \mu_{2}, \ldots, \mu_{n}}\right\| \leq \frac{1}{r\left(L_{\mu_{1}, \mu_{2}, \ldots, \mu_{n}}\right)} \int_{0}^{1}\left[\mu_{n} K_{n}(1, s)\right. \\
& \left.+\mu_{n-1} K_{n-1}(1, s)+\cdots+\mu_{1} K_{1}(1, s)\right] \\
& \cdot \varphi_{\mu_{1}, \mu_{2}, \ldots, \mu_{n}}(s) d s,
\end{aligned}
$$

and

$$
\begin{gathered}
\varphi_{\mu_{1}, \mu_{2}, \ldots, \mu_{n}}(t) \geq \frac{t^{\alpha-\beta_{n-1}-1}}{r\left(L_{\mu_{1}, \mu_{2}, \ldots, \mu_{n}}\right)} \int_{0}^{1}\left[\mu_{n} K_{n}(1, s)\right. \\
\left.+\mu_{n-1} K_{n-1}(1, s)+\cdots+\mu_{1} K_{1}(1, s)\right] \\
\cdot \varphi_{\mu_{1}, \mu_{2}, \ldots, \mu_{n}}(s) d s \geq t^{\alpha-\beta_{n-1}-1}\left\|\varphi_{\mu_{1}, \mu_{2}, \ldots, \mu_{n}}\right\| .
\end{gathered}
$$

This implies

$$
\varphi_{\mu_{1}, \mu_{2}, \ldots, \mu_{n}} \in P_{0}
$$

Lemma 7 (see [44]). Let $E$ be a Banach space and $\Omega$ a bounded open set in E. Suppose that $A: \Omega \longrightarrow E$ is a continuous compact operator. If there exists $u_{0} \in E \backslash\{0\}$ such that 


$$
u-A u \neq \mu u_{0}, \quad \forall u \in \partial \Omega, \quad \mu \geqslant 0,
$$

then the topological degree $\operatorname{deg}(I-A, \Omega, 0)=0$.

Lemma 8 (see [44]). Let $E$ be a Banach space and $\Omega$ a bounded open set in $E$ with $0 \in \Omega$. Suppose that $A: \Omega \longrightarrow E$ is a continuous compact operator. If

$$
A u \neq \mu u, \quad \forall u \in \partial \Omega, \mu \geqslant 1
$$

then the topological degree $\operatorname{deg}(I-A, \Omega, 0)=1$.

\section{Main Results}

Let $\mu_{1}, \mu_{2}, \ldots, \mu_{n} ; \tilde{\mu}_{1}, \tilde{\mu}_{2}, \ldots, \tilde{\mu}_{n}$ be two families of nonnegative real numbers which are not all zero. Now we list our assumptions for $f$ as follows:

(H1) $f:[0,1] \times \mathbb{R}^{n} \longrightarrow \mathbb{R}$ is continuous,

(H2) there exist $b(t), c(t) \in C[0,1]$ with $b(t), c(t) \geq 0(\not \equiv$ $0)$ and $K\left(x_{n}, x_{n-1}, \ldots, x_{1}\right) \in C\left[\mathbb{R}^{n}, \mathbb{R}^{+}\right]$such that

$$
\begin{aligned}
f\left(t, x_{n}, x_{n-1}, \ldots, x_{1}\right) \geqslant & -b(t) \\
& -c(t) K\left(x_{n}, x_{n-1}, \ldots, x_{1}\right),
\end{aligned}
$$

$\forall x_{i} \in \mathbb{R}, t \in[0,1], i=1,2, \ldots, n$,

(H3) $\lim _{\mu_{n}\left|x_{n}\right|+\mu_{n-1}\left|x_{n-1}\right|+\cdots+\mu_{1}\left|x_{1}\right| \longrightarrow+\infty}\left(K\left(x_{n}, x_{n-1}, \ldots, x_{1}\right) /\right.$ $\left.\left(\mu_{n}\left|x_{n}\right|+\mu_{n-1}\left|x_{n-1}\right|+\cdots+\mu_{1}\left|x_{1}\right|\right)\right)=0$,

(H4) $\liminf \mu_{\mu_{n}\left|x_{n}\right|+\mu_{n-1}\left|x_{n-1}\right|+\cdots+\mu_{1}\left|x_{1}\right| \rightarrow+\infty}\left(f\left(t, x_{n}, x_{n-1}, \ldots\right.\right.$, $\left.\left.x_{1}\right) /\left(\mu_{n}\left|x_{n}\right|+\mu_{n-1}\left|x_{n-1}\right|+\cdots+\mu_{1}\left|x_{1}\right|\right)\right)>\lambda_{\mu_{1}, \mu_{2}, \ldots, \mu_{n}}$, uniformly for $t \in[0,1]$,

(H5) $\lim \sup _{\widetilde{\mu}_{n}\left|x_{n}\right|+\widetilde{\mu}_{n-1}\left|x_{n-1}\right|+\cdots+\widetilde{\mu}_{1}\left|x_{1}\right| \rightarrow 0}\left(\mid\left(f\left(t, x_{n}, x_{n-1}, \ldots\right.\right.\right.$, $\left.\left.x_{1}\right) \mid /\left(\widetilde{\mu}_{n}\left|x_{n}\right|+\widetilde{\mu}_{n-1}\left|x_{n-1}\right|+\cdots+\widetilde{\mu}_{1}\left|x_{1}\right|\right)\right)<\lambda_{\widetilde{\mu}_{1}, \widetilde{\mu}_{2}, \ldots, \widetilde{\mu}_{n}}$, uniformly for $t \in[0,1]$, where

$$
\begin{aligned}
& \lambda_{\mu_{1}, \mu_{2}, \ldots, \mu_{n}}=r^{-1}\left(L_{\mu_{1}, \mu_{2}, \ldots, \mu_{n}}\right), \\
& \lambda_{\widetilde{\mu}_{1}, \widetilde{\mu}_{2}, \ldots, \widetilde{\mu}_{n}}=r^{-1}\left(L_{\widetilde{\mu}_{1}, \widetilde{\mu}_{2}, \ldots, \widetilde{\mu}_{n}}\right) .
\end{aligned}
$$

Theorem 9. Assume that (H1)-(H5) are satisfied. Then (1) has at least one nontrivial solution.

Proof. From (H4), there exist $\varepsilon_{0}>0$ and $X_{0}>0$ such that

$$
\begin{array}{r}
f\left(t, x_{n}, x_{n-1}, \ldots, x_{1}\right) \geqslant\left(\lambda_{\mu_{1}, \mu_{2}, \ldots, \mu_{n}}+\varepsilon_{0}\right) \\
\cdot\left(\mu_{n}\left|x_{n}\right|+\mu_{n-1}\left|x_{n-1}\right|+\cdots+\mu_{1}\left|x_{1}\right|\right),
\end{array}
$$

for all $t \in[0,1], \mu_{n}\left|x_{n}\right|+\mu_{n-1}\left|x_{n-1}\right|+\cdots+\mu_{1}\left|x_{1}\right|>X_{0}$.
For any given $\varepsilon$ with $\varepsilon_{0}-\|c\| \varepsilon>0$, and using (H3) there exists $X_{1}>X_{0}$ such that

$$
\begin{aligned}
& K\left(x_{n}, x_{n-1}, \ldots, x_{1}\right) \\
& \leqslant \varepsilon\left(\mu_{n}\left|x_{n}\right|+\mu_{n-1}\left|x_{n-1}\right|+\cdots+\mu_{1}\left|x_{1}\right|\right), \\
& \quad \forall \mu_{n}\left|x_{n}\right|+\mu_{n-1}\left|x_{n-1}\right|+\cdots+\mu_{1}\left|x_{1}\right|>X_{1} .
\end{aligned}
$$

Hence, (H2), (35), and (36) enable us to obtain

$$
\begin{aligned}
f & \left(t, x_{n}, x_{n-1}, \ldots, x_{1}\right) \geqslant\left(\lambda_{\mu_{1}, \mu_{2}, \ldots, \mu_{n}}+\varepsilon_{0}\right) \\
& \cdot\left(\mu_{n}\left|x_{n}\right|+\mu_{n-1}\left|x_{n-1}\right|+\cdots+\mu_{1}\left|x_{1}\right|\right)-b(t) \\
& -c(t) K\left(x_{n}, x_{n-1}, \ldots, x_{1}\right) \geqslant\left(\lambda_{\mu_{1}, \mu_{2}, \ldots, \mu_{n}}+\varepsilon_{0}\right) \\
& \cdot\left(\mu_{n}\left|x_{n}\right|+\mu_{n-1}\left|x_{n-1}\right|+\cdots+\mu_{1}\left|x_{1}\right|\right)-b(t) \\
& -\varepsilon c(t)\left(\mu_{n}\left|x_{n}\right|+\mu_{n-1}\left|x_{n-1}\right|+\cdots+\mu_{1}\left|x_{1}\right|\right) \\
& \geqslant\left(\lambda_{\mu_{1}, \mu_{2}, \ldots, \mu_{n}}+\varepsilon_{0}-\varepsilon c(t)\right) \\
& \cdot\left(\mu_{n}\left|x_{n}\right|+\mu_{n-1}\left|x_{n-1}\right|+\cdots+\mu_{1}\left|x_{1}\right|\right)-b(t) \\
& \geqslant\left(\lambda_{\mu_{1}, \mu_{2}, \ldots, \mu_{n}}+\varepsilon_{0}-\varepsilon\|c\|\right) \\
& \cdot\left(\mu_{n}\left|x_{n}\right|+\mu_{n-1}\left|x_{n-1}\right|+\cdots+\mu_{1}\left|x_{1}\right|\right)-\|b\|
\end{aligned}
$$

for all $t \in[0,1], \mu_{n}\left|x_{n}\right|+\mu_{n-1}\left|x_{n-1}\right|+\cdots+\mu_{1}\left|x_{1}\right|>X_{1}$.

Let

$$
\begin{aligned}
& C_{X_{1}}=\left(\lambda_{\mu_{1}, \mu_{2}, \ldots, \mu_{n}}+\varepsilon_{0}-\varepsilon\|c\|\right) X_{1} \\
& \quad+\max _{0 \leqslant t \leqslant 1, \mu_{n}\left|x_{n}\right|+\mu_{n-1}\left|x_{n-1}\right|+\cdots+\mu_{1}\left|x_{1}\right| \leqslant X_{1}} \mid f\left(t, x_{n}, x_{n-1}, \ldots,\right. \\
& \left.x_{1}\right) \mid, \\
& K^{*}=\max _{\mu_{n}\left|x_{n}\right|+\mu_{n-1}\left|x_{n-1}\right|+\cdots+\mu_{1}\left|x_{1}\right| \leqslant X_{1}} K\left(x_{n}, x_{n-1}, \ldots, x_{1}\right) .
\end{aligned}
$$

Then we obtain

$$
\begin{aligned}
& f\left(t, x_{n}, x_{n-1}, \ldots, x_{1}\right) \geqslant\left(\lambda_{\mu_{1}, \mu_{2}, \ldots, \mu_{n}}+\varepsilon_{0}-\varepsilon\|c\|\right) \\
& \cdot\left(\mu_{n}\left|x_{n}\right|+\mu_{n-1}\left|x_{n-1}\right|+\cdots+\mu_{1}\left|x_{1}\right|\right)-b(t) \\
& -C_{X_{1}},
\end{aligned}
$$

for all $\left(t, x_{n}, x_{n-1}, \ldots, x_{1}\right) \in[0,1] \times \mathbb{R}^{n}$. Note that $\varepsilon$ can be chosen arbitrarily small, and let

$$
\begin{aligned}
R>\max \left\{\frac{\|b\|+\|c\| K^{*}+C_{X_{1}}}{(\alpha-\beta)\left(\Gamma\left(\alpha-\beta_{n-1}\right)-\varepsilon M_{\mu_{1}, \mu_{2}, \ldots, \mu_{n}}\|c\|\right)},\right. \\
\\
\left.\quad \frac{\left(\lambda_{\mu_{1}, \mu_{2}, \ldots, \mu_{n}}+2 \varepsilon_{0}-2\|c\| \varepsilon\right)\left(\|b\|+\|c\| K^{*}+C_{X_{1}}\right)}{(\alpha-\beta) \Gamma\left(\alpha-\beta_{n-1}\right)\left(\varepsilon_{0}-\|c\| \varepsilon-\left(\lambda_{\mu_{1}, \mu_{2}, \ldots, \mu_{n}}+2 \varepsilon_{0}-2\|c\| \varepsilon\right)\left(\varepsilon\|c\| M_{\mu_{1}, \mu_{2}, \ldots, \mu_{n}} / \Gamma\left(\alpha-\beta_{n-1}\right)\right)\right)}\right\},
\end{aligned}
$$


6

Journal of Function Spaces

where $M_{\mu_{1}, \mu_{2}, \ldots, \mu_{n}}=\int_{0}^{1}(1-s)^{\alpha-\beta-1}\left(\left(\mu_{n} / \Gamma\left(\beta_{n-1}\right)\right) \int_{0}^{s}(s-\right.$ $\left.\tau)^{\beta_{n-1}-1} d \tau+\left(\mu_{n-1} / \Gamma\left(\beta_{n-1}-\beta_{1}\right)\right) \int_{0}^{s}(s-\tau)^{\beta_{n-1}-\beta_{1}-1} d \tau+\cdots+\mu_{1}\right) d s$.

Now we claim

$$
v-A v \neq \mu \varphi_{\mu_{1}, \mu_{2}, \ldots, \mu_{n}}, \quad \forall v \in \partial B_{R}, \quad \mu \geqslant 0,
$$

where $\varphi_{\mu_{1}, \mu_{2}, \ldots, \mu_{n}}$ is the positive eigenfunction of $L_{\mu_{1}, \mu_{2}, \ldots, \mu_{n}}$ corresponding to the eigenvalue $\lambda_{\mu_{1}, \mu_{2}, \ldots, \mu_{n}}$, ie., $\varphi_{\mu_{1}, \mu_{2}, \ldots, \mu_{n}}=$ $\lambda_{\mu_{1}, \mu_{2}, \ldots, \mu_{n}} L_{\mu_{1}, \mu_{2}, \ldots, \mu_{n}} \varphi_{\mu_{1}, \mu_{2}, \ldots, \mu_{n}}$, and from $(30), \varphi_{\mu_{1}, \mu_{2}, \ldots, \mu_{n}} \in P_{0}$.

Suppose (41) is false. Then there exist $v_{0} \in \partial B_{R}$ and $\mu_{0}>0$ such that

$$
v_{0}-A v_{0}=\mu_{0} \varphi_{\mu_{1}, \mu_{2}, \ldots, \mu_{n}} .
$$

Let

$$
\begin{array}{r}
\widetilde{v}(t)=\int_{0}^{1} K_{1}(t, s)\left[b(s)+c(s) K\left(I_{0+}^{\beta_{n-1}} v_{0}(s),\right.\right. \\
\left.\left.I_{0+}^{\beta_{n-1}-\beta_{1}} v_{0}(s), \ldots, I_{0+}^{\beta_{n-1}-\beta_{n-2}} v_{0}(s), v_{0}(s)\right)+C_{X_{1}}\right] d s \\
\forall t \in[0,1] .
\end{array}
$$

Then we have

$$
\begin{aligned}
& \widetilde{v}(t) \\
& \leqslant \int_{0}^{1} K_{1}(t, s)\left[b(s)+c(s)\left[\varepsilon \left(\mu_{n}\left|I_{0+}^{\beta_{n-1}} v_{0}(s)\right|\right.\right.\right. \\
& \left.\left.+\mu_{n-1}\left|I_{0+}^{\beta_{n-1}-\beta_{1}} v_{0}(s)\right|+\cdots+\mu_{1}\left|v_{0}(s)\right|\right)+K^{*}\right] \\
& \left.+C_{X_{1}}\right] d s \\
& \leqslant \frac{t^{\alpha-\beta_{n-1}-1}}{\Gamma\left(\alpha-\beta_{n-1}\right)} \int_{0}^{1}(1 \\
& -s)^{\alpha-\beta-1}\left[b(s)+c(s)\left[\varepsilon \left(\frac{\mu_{n}}{\Gamma\left(\beta_{n-1}\right)}\right.\right.\right. \\
& \cdot \int_{0}^{s}(s-\tau)^{\beta_{n-1}-1}\left|v_{0}(\tau)\right| d \tau+\frac{\mu_{n-1}}{\Gamma\left(\beta_{n-1}-\beta_{1}\right)} \\
& \left.\cdot \int_{0}^{s}(s-\tau)^{\beta_{n-1}-\beta_{1}-1}\left|v_{0}(\tau)\right| d \tau+\cdots+\mu_{1}\left|v_{0}(s)\right|\right) \\
& \left.\left.+K^{*}\right]+C_{X_{1}}\right] d s \\
& \leq \frac{t^{\alpha-\beta_{n-1}-1}}{\Gamma\left(\alpha-\beta_{n-1}\right)} \int_{0}^{1}(1 \\
& -s)^{\alpha-\beta-1}\left[b(s)+c(s)\left[\varepsilon \left(\frac{\mu_{n}}{\Gamma\left(\beta_{n-1}\right)}\right.\right.\right. \\
& \cdot \int_{0}^{s}(s-\tau)^{\beta_{n-1}-1} d \tau+\frac{\mu_{n-1}}{\Gamma\left(\beta_{n-1}-\beta_{1}\right)}
\end{aligned}
$$

$$
\begin{aligned}
& \left.\left.\cdot \int_{0}^{s}(s-\tau)^{\beta_{n-1}-\beta_{1}-1} d \tau+\cdots+\mu_{1}\right)\left\|v_{0}\right\|+K^{*}\right] \\
& \left.+C_{X_{1}}\right] d s \leqslant \frac{t^{\alpha-\beta_{n-1}-1}\left(\|b\|+\|c\| K^{*}+C_{X_{1}}\right)}{(\alpha-\beta) \Gamma\left(\alpha-\beta_{n-1}\right)}
\end{aligned}
$$

$$
\begin{aligned}
& +\frac{t^{\alpha-\beta_{n-1}-1}}{\Gamma\left(\alpha-\beta_{n-1}\right)} \varepsilon\|c\| \int_{0}^{1}(1 \\
& -s)^{\alpha-\beta-1}\left(\frac{\mu_{n}}{\Gamma\left(\beta_{n-1}\right)} \int_{0}^{s}(s-\tau)^{\beta_{n-1}-1} d \tau\right. \\
& \left.+\frac{\mu_{n-1}}{\Gamma\left(\beta_{n-1}-\beta_{1}\right)} \int_{0}^{s}(s-\tau)^{\beta_{n-1}-\beta_{1}-1} d \tau+\cdots+\mu_{1}\right) \\
& \cdot\left\|v_{0}\right\| d s \leq \frac{\|b\|+\|c\| K^{*}+C_{X_{1}}}{(\alpha-\beta) \Gamma\left(\alpha-\beta_{n-1}\right)} \\
& +\frac{\varepsilon\|c\| R}{\Gamma\left(\alpha-\beta_{n-1}\right)} \int_{0}^{1}(1 \\
& +s)^{\alpha-\beta-1}\left(\frac{\mu_{n}}{\Gamma\left(\beta_{n-1}\right)} \int_{0}^{s}(s-\tau)^{\beta_{n-1}-1} d \tau\right. \\
& +\frac{\mu_{n-1}}{\Gamma\left(\beta_{n-1}-\beta_{1}\right)} \int_{0}^{s}(s-\tau)^{\beta_{n-1}-\beta_{1}-1} d \tau+\cdots \\
& \left.\mu_{1}\right) d s .
\end{aligned}
$$

Consequently, we have

$$
\begin{aligned}
\|\widetilde{v}\| & \leqslant \frac{\|b\|+\|c\| K^{*}+C_{X_{1}}}{(\alpha-\beta) \Gamma\left(\alpha-\beta_{n-1}\right)}+\frac{\varepsilon\|c\| R}{\Gamma\left(\alpha-\beta_{n-1}\right)} M_{\mu_{1}, \mu_{2}, \ldots, \mu_{n}} \\
& <R .
\end{aligned}
$$

Note from (43) and (25), $\widetilde{v} \in P_{0}$, and then $\varphi_{\mu_{1}, \mu_{2}, \ldots, \mu_{n}} \in P_{0}$ implies that

$$
\begin{aligned}
& v_{0}(t)+\widetilde{v}(t)=A v_{0}(t)+\mu_{0} \varphi_{\mu_{1}, \mu_{2}, \ldots, \mu_{n}}(t)+\widetilde{v}(t) \\
& =\int_{0}^{1} K_{1}(t, s)\left[f \left(s, I_{0+}^{\beta_{n-1}} v_{0}(s), I_{0+}^{\beta_{n-1}-\beta_{1}} v_{0}(s), \ldots,\right.\right. \\
& \left.I_{0+}^{\beta_{n-1}-\beta_{n-2}} v_{0}(s), v_{0}(s)\right)+b(s)+c(s) K\left(I_{0+}^{\beta_{n-1}} v_{0}(s),\right. \\
& \left.\left.I_{0+}^{\beta_{n-1}-\beta_{1}} v_{0}(s), \ldots, I_{0+}^{\beta_{n-1}-\beta_{n-2}} v_{0}(s), v_{0}(s)\right)+C_{X_{1}}\right] d s \\
& +\mu_{0} \varphi_{\mu_{1}, \mu_{2}, \ldots, \mu_{n}}(t) .
\end{aligned}
$$

Then we have

$$
v_{0}+\widetilde{v} \in P_{0}
$$


Therefore, (26), (39), and (43) enable us to obtain

$$
\begin{aligned}
& A v_{0}(t)+\widetilde{v}(t)=\int_{0}^{1} K_{1}(t, s) f\left(s, I_{0+}^{\beta_{n-1}} v_{0}(s),\right. \\
& \left.I_{0+}^{\beta_{n-1}-\beta_{1}} v_{0}(s), \ldots, I_{0+}^{\beta_{n-1}-\beta_{n-2}} v_{0}(s), v_{0}(s)\right) d s \\
& +\int_{0}^{1} K_{1}(t, s)\left[b(s)+c(s) K\left(I_{0+}^{\beta_{n-1}} v_{0}(s),\right.\right. \\
& \left.\left.I_{0+}^{\beta_{n-1}-\beta_{1}} v_{0}(s), \ldots, I_{0+}^{\beta_{n-1}-\beta_{n-2}} v_{0}(s), v_{0}(s)\right)+C_{X_{1}}\right] d s \\
& \geqslant\left(\lambda_{\mu_{1}, \mu_{2}, \ldots, \mu_{n}}+\varepsilon_{0}-\varepsilon\|c\|\right) \int_{0}^{1} K_{1}(t, s) \\
& \cdot\left[\mu_{n}\left|I_{0+}^{\beta_{n-1}} v_{0}(s)\right|+\mu_{n-1}\left|I_{0+}^{\beta_{n-1}-\beta_{1}} v_{0}(s)\right|+\cdots\right. \\
& \left.+\mu_{1}\left|v_{0}(s)\right|\right] d s-\int_{0}^{1} K_{1}(t, s)\left[b(s)+C_{X_{1}}\right] d s \\
& +\int_{0}^{1} K_{1}(t, s)\left[b(s)+c(s) K\left(I_{0+}^{\beta_{n-1}} v_{0}(s),\right.\right. \\
& \left.\left.I_{0+}^{\beta_{n-1}-\beta_{1}} v_{0}(s), \ldots, I_{0+}^{\beta_{n-1}-\beta_{n-2}} v_{0}(s), v_{0}(s)\right)+C_{X_{1}}\right] d s \\
& \geqslant\left(\lambda_{\mu_{1}, \mu_{2}, \ldots, \mu_{n}}+\varepsilon_{0}-\varepsilon\|c\|\right) \int_{0}^{1} K_{1}(t, s) \\
& \cdot\left[\frac{\mu_{n}}{\Gamma\left(\beta_{n-1}\right)}\left|\int_{0}^{s}(s-\tau)^{\beta_{n-1}-1} v_{0}(\tau) d \tau\right|\right. \\
& +\frac{\mu_{n-1}}{\Gamma\left(\beta_{n-1}-\beta_{1}\right)}\left|\int_{0}^{s}(s-\tau)^{\beta_{n-1}-\beta_{1}-1} v_{0}(\tau) d \tau\right|+\cdots \\
& \left.+\mu_{1}\left|v_{0}(s)\right|\right] d s \geqslant\left(\lambda_{\mu_{1}, \mu_{2}, \ldots, \mu_{n}}+\varepsilon_{0}-\varepsilon\|c\|\right) \\
& \cdot \mid \frac{\mu_{n}}{\Gamma\left(\beta_{n-1}\right)} \int_{0}^{1} K_{1}(t, s) \int_{0}^{s}(s-\tau)^{\beta_{n-1}-1} v_{0}(\tau) d \tau d s \\
& +\frac{\mu_{n-1}}{\Gamma\left(\beta_{n-1}-\beta_{1}\right)} \int_{0}^{1} K_{1}(t, s) \int_{0}^{s}(s-\tau)^{\beta_{n-1}-\beta_{1}-1} \\
& \cdot v_{0}(\tau) d \tau d s+\cdots+\mu_{1} \int_{0}^{1} K_{1}(t, s) v_{0}(s) d s \\
& \geqslant\left(\lambda_{\mu_{1}, \mu_{2}, \ldots, \mu_{n}}+\varepsilon_{0}-\varepsilon\|c\|\right) \mid \mu_{n} \int_{0}^{1} K_{n}(t, \tau) v_{0}(\tau) d \tau \\
& +\mu_{n-1} \int_{0}^{1} K_{n-1}(t, \tau) v_{0}(\tau) d \tau+\cdots+\mu_{1} \int_{0}^{1} K_{1}(t \\
& \text { s) } v_{0}(s) d s \mid=\left(\lambda_{\mu_{1}, \mu_{2}, \ldots, \mu_{n}}+\varepsilon_{0}-\varepsilon\|c\|\right) \\
& \cdot\left|\int_{0}^{1} K_{\mu_{1}, \mu_{2}, \ldots, \mu_{n}}(t, s) v_{0}(s) d s\right| \geqslant\left(\lambda_{\mu_{1}, \mu_{2}, \ldots, \mu_{n}}+\varepsilon_{0}\right. \\
& -\varepsilon\|c\|) \int_{0}^{1} K_{\mu_{1}, \mu_{2}, \ldots, \mu_{n}}(t, s) v_{0}(s) d s .
\end{aligned}
$$

Consequently, we obtain

$$
\begin{aligned}
& \left(\lambda_{\mu_{1}, \mu_{2}, \ldots, \mu_{n}}+\varepsilon_{0}-\|c\| \varepsilon\right) \int_{0}^{1} K_{\mu_{1}, \mu_{2}, \ldots, \mu_{n}}(t, s) v_{0}(s) d s \\
& \quad=\lambda_{\mu_{1}, \mu_{2}, \ldots, \mu_{n}} \int_{0}^{1} K_{\mu_{1}, \mu_{2}, \ldots, \mu_{n}}(t, s)\left(v_{0}(s)+\widetilde{v}(s)\right) d s \\
& +\left(\varepsilon_{0}-\|c\| \varepsilon\right) \int_{0}^{1} K_{\mu_{1}, \mu_{2}, \ldots, \mu_{n}}(t, s) v_{0}(s) d s \\
& -\lambda_{\mu_{1}, \mu_{2}, \ldots, \mu_{n}} \int_{0}^{1} K_{\mu_{1}, \mu_{2}, \ldots, \mu_{n}}(t, s) \widetilde{v}(s) d s \\
& \quad=\lambda_{\mu_{1}, \mu_{2}, \ldots, \mu_{n}} L_{\mu_{1}, \mu_{2}, \ldots, \mu_{n}}\left(v_{0}+\widetilde{v}\right)(t)+\left(\varepsilon_{0}-\|c\| \varepsilon\right) \\
& \quad \cdot \int_{0}^{1} K_{\mu_{1}, \mu_{2}, \ldots, \mu_{n}}(t, s)\left(v_{0}(s)+\widetilde{v}(s)\right) d s \\
& \quad-\left(\lambda_{\mu_{1}, \mu_{2}, \ldots, \mu_{n}}+\varepsilon_{0}-\|c\| \varepsilon\right) \\
& \quad \cdot \int_{0}^{1} K_{\mu_{1}, \mu_{2}, \ldots, \mu_{n}}(t, s) \widetilde{v}(s) d s .
\end{aligned}
$$

From (47), $v_{0}(t)+\widetilde{v}(t) \geqslant t^{\alpha-\beta_{n-1}-1}\left\|v_{0}+\widetilde{v}\right\| \geqslant t^{\alpha-\beta_{n-1}-1}\left(\left\|v_{0}\right\|-\right.$ $\|\widetilde{v}\|), t \in[0,1]$, and from (44), we have

$$
\begin{aligned}
& \left(\varepsilon_{0}-\|c\| \varepsilon\right) \int_{0}^{1} K_{\mu_{1}, \mu_{2}, \ldots, \mu_{n}}(t, s)\left(v_{0}(s)+\widetilde{v}(s)\right) d s \\
& -\left(\lambda_{\mu_{1}, \mu_{2}, \ldots, \mu_{n}}+\varepsilon_{0}-\|c\| \varepsilon\right) \\
& \cdot \int_{0}^{1} K_{\mu_{1}, \mu_{2}, \ldots, \mu_{n}}(t, s) \widetilde{v}(s) d s \geqslant\left(\varepsilon_{0}-\|c\| \varepsilon\right)(R \\
& -\|\widetilde{v}\|) \int_{0}^{1} s^{\alpha-\beta_{n-1}-1} K_{\mu_{1}, \mu_{2}, \ldots, \mu_{n}}(t, s) d s-\left(\lambda_{\mu_{1}, \mu_{2}, \ldots, \mu_{n}}\right. \\
& \left.+\varepsilon_{0}-\|c\| \varepsilon\right) \times\left[\frac{\|b\|+\|c\| K^{*}+C_{X_{1}}}{(\alpha-\beta) \Gamma\left(\alpha-\beta_{n-1}\right)}\right. \\
& \left.+\frac{\varepsilon\|c\| R}{\Gamma\left(\alpha-\beta_{n-1}\right)} M_{\mu_{1}, \mu_{2}, \ldots, \mu_{n}}\right] \\
& \cdot \int_{0}^{1} s^{\alpha-\beta_{n-1}-1} K_{\mu_{1}, \mu_{2}, \ldots, \mu_{n}}(t, s) d s \geqslant 0 .
\end{aligned}
$$

Now from (48), (49), and (50), we find

$$
\begin{aligned}
A v_{0}(t)+\widetilde{v}(t) \geqslant \lambda_{\mu_{1}, \mu_{2}, \ldots, \mu_{n}} L_{\mu_{1}, \mu_{2}, \ldots, \mu_{n}}\left(v_{0}+\widetilde{v}\right)(t), & \\
& t \in[0,1] .
\end{aligned}
$$

Therefore, using (42) and (51), we have

$$
\begin{aligned}
v_{0}+\widetilde{v} & =A v_{0}+\mu_{0} \varphi_{\mu_{1}, \mu_{2}, \ldots, \mu_{n}}+\widetilde{v} \\
& \geqslant \lambda_{\mu_{1}, \mu_{2}, \ldots, \mu_{n}} L_{\mu_{1}, \mu_{2}, \ldots, \mu_{n}}\left(v_{0}+\widetilde{v}\right)+\mu_{0} \varphi_{\mu_{1}, \mu_{2}, \ldots, \mu_{n}} \\
& \geqslant \mu_{0} \varphi_{\mu_{1}, \mu_{2}, \ldots, \mu_{n}} .
\end{aligned}
$$


Define $\mu^{*}=\sup \left\{\mu>0: v_{0}+\widetilde{v} \geqslant \mu \varphi_{\mu_{1}, \mu_{2}, \ldots, \mu_{n}}\right\}$. Then we have $\mu^{*} \geqslant \mu_{0}$ and $v_{0}+\widetilde{v} \geqslant \mu^{*} \varphi_{\mu_{1}, \mu_{2}, \ldots, \mu_{n}}$. From $\varphi_{\mu_{1}, \mu_{2}, \ldots, \mu_{n}}=$ $\lambda_{\mu_{1}, \mu_{2}, \ldots, \mu_{n}} L_{\mu_{1}, \mu_{2}, \ldots, \mu_{n}} \varphi_{\mu_{1}, \mu_{2}, \ldots, \mu_{n}}$, we obtain

$$
\begin{aligned}
& \lambda_{\mu_{1}, \mu_{2}, \ldots, \mu_{n}} L_{\mu_{1}, \mu_{2}, \ldots, \mu_{n}}\left(v_{0}+\widetilde{v}\right) \\
& \quad \geqslant \lambda_{\mu_{1}, \mu_{2}, \ldots, \mu_{n}} L_{\mu_{1}, \mu_{2}, \ldots, \mu_{n}} \mu^{*} \varphi_{\mu_{1}, \mu_{2}, \ldots, \mu_{n}}=\mu^{*} \varphi_{\mu_{1}, \mu_{2}, \ldots, \mu_{n}} .
\end{aligned}
$$

Hence

$$
\begin{aligned}
v_{0}+\widetilde{v} & \geqslant \lambda_{\mu_{1}, \mu_{2}, \ldots, \mu_{n}} L_{\mu_{1}, \mu_{2}, \ldots, \mu_{n}}\left(v_{0}+\widetilde{v}\right)+\mu_{0} \varphi_{\mu_{1}, \mu_{2}, \ldots, \mu_{n}} \\
& \geqslant\left(\mu_{0}+\mu^{*}\right) \varphi_{\mu_{1}, \mu_{2}, \ldots, \mu_{n}},
\end{aligned}
$$

which contradicts the definition of $\mu^{*}$. Therefore, (41) holds, and from Lemma 7, we obtain

$$
\operatorname{deg}\left(I-A, B_{R}, 0\right)=0 .
$$

From (H5), there exist $0<\varepsilon_{1}<\lambda_{\widetilde{\mu}_{1}, \widetilde{\mu}_{2}, \ldots, \widetilde{\mu}_{n}}$ and $0<r<R$ such that

$$
\begin{gathered}
\mid\left(f\left(t, x_{n}, x_{n-1}, \ldots, x_{1}\right) \mid \leqslant\left(\lambda_{\widetilde{\mu}_{1}, \tilde{\mu}_{2}, \ldots, \widetilde{\mu}_{n}}-\varepsilon_{1}\right)\right. \\
\cdot\left(\widetilde{\mu}_{n}\left|x_{n}\right|+\widetilde{\mu}_{n-1}\left|x_{n-1}\right|+\cdots+\widetilde{\mu}_{1}\left|x_{1}\right|\right),
\end{gathered}
$$

for all $x_{i} \in \mathbb{R}, i=1,2, \ldots, n, t \in[0,1]$ with $0 \leqslant \widetilde{\mu}_{n}\left|x_{n}\right|+$ $\widetilde{\mu}_{n-1}\left|x_{n-1}\right|+\cdots+\widetilde{\mu}_{1}\left|x_{1}\right|<r$. Consequently, we obtain

$$
\begin{aligned}
& |(A v)(t)| \leqslant\left(\lambda_{\tilde{\mu}_{1}, \tilde{\mu}_{2}, \ldots, \tilde{\mu}_{n}}-\varepsilon_{1}\right) \int_{0}^{1} K_{1}(t, s)\left(\tilde{\mu}_{n}\left|I_{0+}^{\beta_{n-1}} v(s)\right|\right. \\
& \left.+\widetilde{\mu}_{n-1}\left|I_{0+}^{\beta_{n-1}-\beta_{1}} v(s)\right|+\cdots+\widetilde{\mu}_{1}|v(s)|\right) d s \\
& \leq\left(\lambda_{\widetilde{\mu}_{1}, \tilde{\mu}_{2}, \ldots, \tilde{\mu}_{n}}-\varepsilon_{1}\right) \int_{0}^{1} K_{1}(t, s) \\
& \cdot\left(\frac{\tilde{\mu}_{n}}{\Gamma\left(\beta_{n-1}\right)} \int_{0}^{s}(s-\tau)^{\beta_{n-1}-1}|v(\tau)| d \tau\right. \\
& +\frac{\tilde{\mu}_{n-1}}{\Gamma\left(\beta_{n-1}-\beta_{1}\right)} \int_{0}^{s}(s-\tau)^{\beta_{n-1}-\beta_{1}-1}|v(\tau)| d \tau+\cdots \\
& \left.+\widetilde{\mu}_{1}|v(s)|\right) d s=\left(\lambda_{\widetilde{\mu}_{1}, \widetilde{\mu}_{2}, \ldots, \widetilde{\mu}_{n}}-\varepsilon_{1}\right) \\
& \cdot\left[\widetilde{\mu}_{n} \int_{0}^{1} K_{n}(t, \tau)|v(\tau)| d \tau\right. \\
& +\widetilde{\mu}_{n-1} \int_{0}^{1} K_{n-1}(t, \tau)|v(\tau)| d \tau+\cdots \\
& \left.+\widetilde{\mu}_{1} \int_{0}^{1} K_{1}(t, s)|v(s)| d s\right]=\left(\lambda_{\widetilde{\mu}_{1}, \widetilde{\mu}_{2}, \ldots, \widetilde{\mu}_{n}}-\varepsilon_{1}\right) \\
& \cdot \int_{0}^{1} K_{\tilde{\mu}_{1}, \tilde{\mu}_{2}, \ldots, \tilde{\mu}_{n}}(t, s)|v(s)| d s=\left(\lambda_{\widetilde{\mu}_{1}, \tilde{\mu}_{2}, \ldots, \tilde{\mu}_{n}}-\varepsilon_{1}\right) \\
& \cdot\left(L_{\widetilde{\mu}_{1}, \widetilde{\mu}_{2}, \ldots, \widetilde{\mu}_{n}}|v|\right)(t), \quad \forall t \in[0,1], v \in E,\|v\| \leqslant r .
\end{aligned}
$$

Now for this $r$, we claim

$$
A v \neq \lambda v, \quad \forall v \in \partial B_{r}, \lambda \geqslant 1 .
$$

Suppose the contrary. Then there exist $v_{0} \in \partial B_{r}$ and $\lambda_{0} \geqslant 1$ such that $A v_{0}=\lambda_{0} v_{0}$. Let $\omega(t)=\left|v_{0}(t)\right|$. Then $\omega \in \partial B_{r} \cap P$ and

$$
\begin{aligned}
\omega & \leqslant \frac{1}{\lambda_{0}}\left(\lambda_{\widetilde{\mu}_{1}, \tilde{\mu}_{2}, \ldots, \widetilde{\mu}_{n}}-\varepsilon_{1}\right) L_{\widetilde{\mu}_{1}, \tilde{\mu}_{2}, \ldots, \widetilde{\mu}_{n}} \omega \\
& \leqslant\left(\lambda_{\widetilde{\mu}_{1}, \widetilde{\mu}_{2}, \ldots, \tilde{\mu}_{n}}-\varepsilon_{1}\right) L_{\widetilde{\mu}_{1}, \widetilde{\mu}_{2}, \ldots, \tilde{\mu}_{n}} \omega .
\end{aligned}
$$

By induction, we have $\omega \leqslant\left(\lambda_{\widetilde{\mu}_{1}, \widetilde{\mu}_{2}, \ldots, \widetilde{\mu}_{n}}-\varepsilon_{1}\right)^{m} L_{\widetilde{\mu}_{1}, \widetilde{\mu}_{2}, \ldots, \widetilde{\mu}_{n}}^{m} \omega$, for $m=1,2, \cdots$. As a result, we have

$$
\begin{gathered}
\|\omega\| \leqslant\left(\lambda_{\widetilde{\mu}_{1}, \widetilde{\mu}_{2}, \ldots, \widetilde{\mu}_{n}}-\varepsilon_{1}\right)^{m}\left\|L_{\widetilde{\mu}_{1}, \widetilde{\mu}_{2}, \ldots, \tilde{\mu}_{n}}^{m}\right\|\|\omega\|, \\
1 \leqslant\left(\lambda_{\widetilde{\mu}_{1}, \widetilde{\mu}_{2}, \ldots, \widetilde{\mu}_{n}}-\varepsilon_{1}\right)^{m}\left\|L_{\widetilde{\mu}_{1}, \widetilde{\mu}_{2}, \ldots, \widetilde{\mu}_{n}}^{m}\right\| .
\end{gathered}
$$

Therefore, by Gelfand's theorem, we have

$$
\begin{aligned}
& \left(\lambda_{\tilde{\mu}_{1}, \tilde{\mu}_{2}, \ldots, \tilde{\mu}_{n}}-\varepsilon_{1}\right) r\left(L_{\widetilde{\mu}_{1}, \tilde{\mu}_{2}, \ldots, \tilde{\mu}_{n}}\right) \\
& \quad=\left(\lambda_{\widetilde{\mu}_{1}, \tilde{\mu}_{2}, \ldots, \tilde{\mu}_{n}}-\varepsilon_{1}\right) \lim _{m \longrightarrow \infty} \sqrt[n]{\left\|L_{\widetilde{\mu}_{1}, \tilde{\mu}_{2}, \ldots, \tilde{\mu}_{n}}^{m}\right\|} \geqslant 1 .
\end{aligned}
$$

This contradicts

$$
\begin{aligned}
& \left(\lambda_{\widetilde{\mu}_{1}, \widetilde{\mu}_{2}, \ldots, \widetilde{\mu}_{n}}-\varepsilon_{1}\right) r\left(L_{\widetilde{\mu}_{1}, \widetilde{\mu}_{2}, \ldots, \widetilde{\mu}_{n}}\right)=1-\varepsilon_{1} r\left(L_{\widetilde{\mu}_{1}, \widetilde{\mu}_{2}, \ldots, \widetilde{\mu}_{n}}\right) \\
& \quad<1 .
\end{aligned}
$$

Hence (58) holds, and from Lemma 8, we have

$$
\operatorname{deg}\left(I-A, B_{r}, 0\right)=1 .
$$

Now (55) and (63) together imply that

$$
\begin{aligned}
& \operatorname{deg}\left(I-A, B_{R} \backslash \bar{B}_{r}, 0\right) \\
& \quad=\operatorname{deg}\left(I-A, B_{R}, 0\right)-\operatorname{deg}\left(I-A, B_{r}, 0\right)=-1 .
\end{aligned}
$$

Therefore the operator $A$ has at least one fixed point in $B_{R} \backslash$ $\bar{B}_{r}$. Equivalently, (1) has at least one nontrivial solution. This completes the proof.

Example 10. For convenience in our example, we let $\mu_{i}=$ $\tilde{\mu}_{i}, i=1,2, \ldots, n$. Let 


$$
f\left(t, x_{n}, x_{n-1}, \ldots, x_{1}\right)= \begin{cases}\sum_{i=1}^{m}(-1)^{i} \rho_{i}-\left(1+t^{2}\right)\left|\sum_{j=1}^{n} \mu_{j} x_{j}\right|^{1 / 3} \ln \left(\left|\sum_{j=1}^{n} \mu_{j} x_{j}\right|+1\right)-\left(1+t^{2}\right) \ln 2, & \sum_{j=1}^{n} \mu_{j} x_{j} \in(-\infty,-1], \\ \sum_{i=1}^{m} \rho_{i}\left(\sum_{j=1}^{n} \mu_{j} x_{j}\right)^{i}, & \sum_{j=1}^{n} \mu_{j} x_{j} \in[-1,+\infty),\end{cases}
$$

where $m \in \mathbb{N}_{+}, 0<\rho_{1}<\lambda_{\mu_{1}, \mu_{2}, \ldots, \mu_{n}}$, and $\rho_{i} \geq 0, i=$ $2,3, \ldots, m$, with $\sum_{i=1}^{m}(-1)^{i} \rho_{i} \geq 0$. Let $b(t)=(1+$ $\left.t^{2}\right) \ln 2, c(t)=1+t^{2}$, and $K\left(x_{n}, x_{n-1}, \ldots, x_{1}\right)=$ $\left|\sum_{j=1}^{n} \mu_{j} x_{j}\right|^{1 / 3} \ln \left(\left|\sum_{j=1}^{n} \mu_{j} x_{j}\right|+1\right)$. We now show that conditions (H1)-(H5) are satisfied. Indeed, for the function $K$, we obtain

$$
\begin{aligned}
& \lim _{\mu_{n}\left|x_{n}\right|+\mu_{n-1}\left|x_{n-1}\right|+\cdots+\mu_{1}\left|x_{1}\right| \rightarrow+\infty} \frac{\left|\sum_{j=1}^{n} \mu_{j} x_{j}\right|^{1 / 3} \ln \left(\left|\sum_{j=1}^{n} \mu_{j} x_{j}\right|+1\right)}{\mu_{n}\left|x_{n}\right|+\mu_{n-1}\left|x_{n-1}\right|+\cdots+\mu_{1}\left|x_{1}\right|} \\
& \leq \lim _{\mu_{n}\left|x_{n}\right|+\mu_{n-1}\left|x_{n-1}\right|+\cdots+\mu_{1}\left|x_{1}\right| \rightarrow+\infty} \frac{\left(\sum_{j=1}^{n}\left|\mu_{j} x_{j}\right|\right)^{1 / 3} \ln \left(\sum_{j=1}^{n}\left|\mu_{j} x_{j}\right|+1\right)}{\sum_{j=1}^{n} \mu_{j}\left|x_{j}\right|}=0,
\end{aligned}
$$

and hence, (H3) holds. For the function $f$, it is clear that (H1)(H2) hold. Also note we have

$$
\begin{aligned}
& \liminf _{\mu_{n}\left|x_{n}\right|+\mu_{n-1}\left|x_{n-1}\right|+\cdots+\mu_{1}\left|x_{1}\right| \rightarrow+\infty} \frac{\sum_{i=1}^{m} \rho_{i}\left(\sum_{j=1}^{n} \mu_{j} x_{j}\right)^{i}}{\sum_{j=1}^{n} \mu_{j}\left|x_{j}\right|} \\
& =+\infty
\end{aligned}
$$

and

$$
\limsup _{\substack{\mu_{n}\left|x_{n}\right|+\mu_{n-1}\left|x_{n-1}\right|+\cdots+\mu_{1}\left|x_{1}\right| \rightarrow 0 \\<\lambda_{\mu_{1}, \mu_{2}, \ldots, \mu_{n}}}} \frac{\left|\sum_{i=1}^{m} \rho_{i}\left(\sum_{j=1}^{n} \mu_{j} x_{j}\right)^{i}\right|}{\sum_{j=1}^{n} \mu_{j}\left|x_{j}\right|}=\rho_{1}
$$

Then (H4)-(H5) hold. Therefore, all the conditions in Theorem 9 are satisfied, and thus (1) has at least one nontrivial solution.

Now, we consider a special case of this example. Let $n=2$, $\mu_{1}=1, \mu_{2}=1$, and consider the operators

$$
\begin{aligned}
& \left(L_{\mu_{1}} v\right)(t)=\int_{0}^{1} \mu_{1} K_{1}(t, s) v(s) d s, \\
& \left(L_{\mu_{2}} v\right)(t)=\int_{0}^{1} \mu_{2} K_{2}(t, s) v(s) d s,
\end{aligned}
$$

Then for all $m \in \mathbb{N}$, from Lemma 5(ii), we have

$$
\begin{aligned}
& \left\|L_{\mu_{1}}^{m}\right\|=\max _{t \in[0,1]} \underbrace{\int_{0}^{1} \cdots \int_{0}^{1} K_{1}\left(t, s_{1}\right)}_{m} \\
& \cdot K_{1}\left(s_{1}, s_{2}\right) \cdots K_{1}\left(s_{m-1}, s_{m}\right) d s_{1} d s_{2} \cdots d s_{m} \\
& \leq \underbrace{\int_{0}^{1} \cdots \int_{0}^{1} K_{1}\left(1, s_{1}\right)}_{m} \\
& \cdot K_{1}\left(1, s_{2}\right) \cdots K_{1}\left(1, s_{m}\right) d s_{1} d s_{2} \cdots d s_{m} \\
& =\left(\int_{0}^{1} K_{1}(1, s) d s\right)^{m},
\end{aligned}
$$

and so

$$
\lim _{m \rightarrow \infty} \sqrt[m]{\left\|L_{\mu_{1}}^{m}\right\|} \leq \int_{0}^{1} K_{1}(1, s) d s
$$

For all $m \in \mathbb{N}$, from Lemma 5(ii), we have

$$
\begin{aligned}
& \left.\left\|L_{\mu_{1}}^{m}\right\|=\max _{t \in[0,1}\right]_{0}^{1} \cdots \int_{0}^{1} K_{1}\left(t, s_{1}\right) K_{1}\left(s_{1}, s_{2}\right) \cdots K_{1}\left(s_{m-1}, s_{m}\right) d s_{1} d s_{2} \cdots d s_{m} \\
& \geq \max _{t \in[0,1]} \underbrace{1}_{m} \cdots \int_{0}^{1} t^{\alpha-\beta_{1}-1} K_{1}\left(1, s_{1}\right) s_{1}^{\alpha-\beta_{1}-1} K_{1}\left(1, s_{2}\right) \cdots s_{m-1}^{\alpha-\beta_{1}-1} K_{1}\left(1, s_{m}\right) d s_{1} d s_{2} \cdots d s_{m} \\
& =\int_{0}^{1} K_{1}(1, s) d s\left(\int_{0}^{1} K_{1}(1, s) s^{\alpha-\beta_{1}-1} d s\right)^{m-1},
\end{aligned}
$$

and so

$$
\lim _{m \rightarrow \infty} \sqrt[m]{\left\|L_{\mu_{1}}^{m}\right\|} \geq \int_{0}^{1} K_{1}(1, s) s^{\alpha-\beta_{1}-1} d s
$$


From Gelfand's theorem, we obtain that the spectral radius of $L_{\mu_{1}}$, denoted by $r\left(L_{\mu_{1}}\right)$, satisfies

$$
\int_{0}^{1} K_{1}(1, s) s^{\alpha-\beta_{1}-1} d s \leq r\left(L_{\mu_{1}}\right) \leq \int_{0}^{1} K_{1}(1, s) d s .
$$

Note the definition of $K_{2}$, and from Lemma 5(ii), we have

$$
\begin{aligned}
& \frac{1}{\Gamma\left(\beta_{n-1}-\beta_{n-2}\right)} \\
& \quad \cdot \int_{0}^{\tau} t^{\alpha-\beta_{n-1}-1} K_{1}(1, \tau)(\tau-s)^{\beta_{n-1}-\beta_{n-2}-1} d \tau \\
& \quad \leq K_{2}(t, s)=\frac{1}{\Gamma\left(\beta_{n-1}-\beta_{n-2}\right)} \\
& \quad \cdot \int_{0}^{\tau} K_{1}(t, \tau)(\tau-s)^{\beta_{n-1}-\beta_{n-2}-1} d \tau \\
& \quad \leq \frac{1}{\Gamma\left(\beta_{n-1}-\beta_{n-2}\right)} \int_{0}^{\tau} K_{1}(1, \tau)(\tau-s)^{\beta_{n-1}-\beta_{n-2}-1} d \tau
\end{aligned}
$$

Thus $t^{\alpha-\beta_{n-1}-1} K_{2}(1, s) \leq K_{2}(t, s) \leq K_{2}(1, s)$, for $t, s \in[0,1]$. Using a similar method, we have that the spectral radius of $L_{\mu_{2}}$, denoted by $r\left(L_{\mu_{2}}\right)$, satisfies

$$
\int_{0}^{1} K_{2}(1, s) s^{\alpha-\beta_{1}-1} d s \leq r\left(L_{\mu_{2}}\right) \leq \int_{0}^{1} K_{2}(1, s) d s .
$$

Consequently, the spectral radius of the operator $L_{\mu_{1}, \mu_{2}}$ satisfies

$$
\begin{aligned}
& \int_{0}^{1} K_{1}(1, s) s^{\alpha-\beta_{1}-1} d s+\int_{0}^{1} K_{2}(1, s) s^{\alpha-\beta_{1}-1} d s \\
& \quad \leq r\left(L_{\mu_{1}, \mu_{2}}\right) \leq \int_{0}^{1} K_{1}(1, s) d s+\int_{0}^{1} K_{2}(1, s) d s .
\end{aligned}
$$

Thus

$$
\begin{gathered}
\frac{1}{\int_{0}^{1} K_{1}(1, s) s^{\alpha-\beta_{1}-1} d s+\int_{0}^{1} K_{2}(1, s) s^{\alpha-\beta_{1}-1} d s} \geq \lambda_{\mu_{1}, \mu_{2}} \\
\quad \geq \frac{1}{\int_{0}^{1} K_{1}(1, s) d s+\int_{0}^{1} K_{2}(1, s) d s} .
\end{gathered}
$$

Now take $\rho_{1} \in\left(0,1 /\left(\int_{0}^{1} K_{1}(1, s) d s+\int_{0}^{1} K_{2}(1, s) d s\right)\right)$.

\section{Data Availability}

No data were used to support this study.

\section{Conflicts of Interest}

The authors declare that they have no conflicts of interest.

\section{Authors' Contributions}

All authors contributed equally to the writing of this paper. All authors read and approved the final manuscript.

\section{Acknowledgments}

This work is supported by Talent Project of Chongqing Normal University (Grant No. 02030307-0040), the National Natural Science Foundation of China (Grant No. 11601048), Natural Science Foundation of Chongqing (Grant No. cstc2016jcyjA0181), Natural Science Foundation of Chongqing Normal University (Grant No. 16XYY24), and the Natural Science Foundation of Shandong Province (ZR2018MA009, ZR2015AM014).

\section{References}

[1] Y. Wang, L. Liu, and Y. Wu, "Positive solutions for a nonlocal fractional differential equation," Nonlinear Analysis: Theory, Methods \& Applications, vol. 74, no. 11, pp. 3599-3605, 2011.

[2] J. Jiang, W. Liu, and H. Wang, "Positive solutions for higher order nonlocal fractional differential equation with integral boundary conditions," Journal of Function Spaces, vol. 2018, Article ID 6598351, 12 pages, 2018.

[3] X. Liu, L. Liu, and Y. Wu, "Existence of positive solutions for a singular nonlinear fractional differential equation with integral boundary conditions involving fractional derivatives," Boundary Value Problems, Article ID 24, 21 pages, 2018.

[4] X. Zhang, L. Liu, and Y. Wu, "The eigenvalue problem for a singular higher order fractional differential equation involving fractional derivatives," Applied Mathematics and Computation, vol. 218, no. 17, pp. 8526-8536, 2012.

[5] Z. Bai, "On positive solutions of a nonlocal fractional boundary value problem," Nonlinear Analysis: Theory, Methods \& Applications, vol. 72, no. 2, pp. 916-924, 2010.

[6] J. Xu and Z. Wei, "Positive solutions for a class of fractional boundary value problems," Nonlinear Analysis: Modelling and Control, vol. 21, no. 1, pp. 1-17, 2016.

[7] J. Jiang, L. Liu, and Y. Wu, "Positive solutions to singular fractional differential system with coupled boundary conditions," Communications in Nonlinear Science and Numerical Simulation, vol. 18, no. 11, pp. 3061-3074, 2013.

[8] Y. Wang, L. Liu, X. Zhang, and Y. Wu, "Positive solutions of an abstract fractional semipositone differential system model for bioprocesses of HIV infection," Applied Mathematics and Computation, vol. 258, pp. 312-324, 2015.

[9] K. Zhang, J. Wang, and W. Ma, "Solutions for integral boundary value problems of nonlinear Hadamard fractional differential equations," Journal of Function Spaces, vol. 2018, Article ID 2193234, 10 pages, 2018.

[10] Y. Cui, "Uniqueness of solution for boundary value problems for fractional differential equations," Applied Mathematics Letters, vol. 51, pp. 48-54, 2016.

[11] Y. Cui, W. Ma, Q. Sun, and X. Su, "New uniqueness results for boundary value problem of fractional differential equation," Nonlinear Analysis: Modelling and Control, vol. 23, no. 1, pp. 3139, 2018.

[12] Y. Zou and G. He, "On the uniqueness of solutions for a class of fractional differential equations," Applied Mathematics Letters, vol. 74, pp. 68-73, 2017.

[13] Z. Yue and Y. Zou, "New uniqueness results for fractional differential equation with dependence on the first order derivative," Advances in Difference Equations, vol. 2019, no. 1, Article ID 38, 9 pages, 2019. 
[14] K. Zhang and Z. Fu, "Solutions for a class of Hadamard fractional boundary value problems with sign-changing nonlinearity," Journal of Function Spaces, Article ID 9046472, 7 pages, 2019.

[15] S. Zhang, "Positive solutions to singular boundary value problem for nonlinear fractional differential equation," Computers \& Mathematics with Applications, vol. 59, no. 3, pp. 1300-1309, 2010.

[16] L. Guo, L. Liu, and Y. Wu, "Iterative unique positive solutions for singular p-Laplacian fractional differential equation system with several parameters," Nonlinear Analysis: Modelling and Control, vol. 23, no. 2, pp. 182-203, 2018.

[17] Y.-H. Su, Y. Yun, D. Wang, and W. Hu, "Existence of solutions to nonlinear $p$-Laplacian fractional differential equations with higher-order derivative terms," Electronic Journal of Differential Equations, vol. 105, pp. 1-24, 2018.

[18] Y. Zhang, "Existence results for a coupled system of nonlinear fractional multi-point boundary value problems at resonance," Journal of Inequalities and Applications, Article ID 198, 17 pages, 2018.

[19] W. Cheng, J. Xu, and Y. Cui, "Positive solutions for a system of nonlinear semipositone fractional $q$-difference equations with $q$-integral boundary conditions," The Journal of Nonlinear Science and Applications, vol. 10, no. 08, pp. 4430-4440, 2017.

[20] J. Wu, X. Zhang, L. Liu, Y. Wu, and Y. Cui, “The convergence analysis and error estimation for unique solution of a $\mathrm{p}$ Laplacian fractional differential equation with singular decreasing nonlinearity," Boundary Value Problems, vol. 2018, Article ID 82, 15 pages, 2018.

[21] X. Hao, H. Wang, L. Liu, and Y. Cui, "Positive solutions for a system of nonlinear fractional nonlocal boundary value problems with parameters and $p$-Laplacian operator," Boundary Value Problems, vol. 2017, no. 1, Article ID 182, 18 pages, 2017.

[22] M. Zuo, X. Hao, L. Liu, and Y. Cui, "Existence results for impulsive fractional integro-differential equation of mixed type with constant coefficient and antiperiodic boundary conditions," Boundary Value Problems, vol. 2017, Article ID 161, 15 pages, 2017.

[23] X. Zhang, L. Liu, and Y. Zou, "Fixed-point theorems for systems of operator equations and their applications to the fractional differential equations," Journal of Function Spaces, vol. 2018, Article ID 7469868, 9 pages, 2018.

[24] Q. Sun, H. Ji, and Y. Cui, "Positive solutions for boundary value problems of fractional differential equation with integral boundary conditions," Journal of Function Spaces, vol. 2018, Article ID 6461930, 6 pages, 2018.

[25] C. Zhai, W. Wang, and H. Li, "A uniqueness method to a new Hadamard fractional differential system with four-point boundary conditions," Journal of Inequalities and Applications, Article ID 207, 16 pages, 2018.

[26] Q. Song, X. Dong, Z. Bai, and B. Chen, "Existence for fractional Dirichlet boundary value problem under barrier strip conditions," Journal of Nonlinear Sciences and Applications. JNSA, vol. 10, no. 7, pp. 3592-3598, 2017.

[27] X. Zhang, J. Wu, L. Liu, Y. Wu, and Y. Cui, "Convergence analysis of iterative scheme and error estimation of positive solution for a fractional differential equation," Mathematical Modelling and Analysis, vol. 23, no. 4, pp. 611-626, 2018.

[28] J. He, X. Zhang, L. Liu, Y. Wu, and Y. Cui, "Existence and asymptotic analysis of positive solutions for a singular fractional differential equation with nonlocal boundary conditions," Boundary Value Problems, Article ID 189, 17 pages, 2018.
[29] X. Zhang, L. Liu, Y. Wu, and Y. Zou, "Existence and uniqueness of solutions for systems of fractional differential equations with Riemann-Stieltjes integral boundary condition," Advances in Difference Equations, vol. 2018, Article ID 204, 15 pages, 2018.

[30] Y. Guo, "Nontrivial solutions for boundary-value problems of nonlinear fractional differential equations," Bulletin of the Korean Mathematical Society, vol. 47, no. 1, pp. 81-87, 2010.

[31] W. Ma, S. Meng, and Y. Cui, "Resonant integral boundary value problems for caputo fractional differential equations," Mathematical Problems in Engineering, vol. 2018, Article ID 5438592, 8 pages, 2018.

[32] W. Ma and Y. Cui, "The eigenvalue problem for Caputo type fractional differential equation with Riemann-Stieltjes integral boundary conditions," Journal of Function Spaces, vol. 2018, Article ID 2176809, 9 pages, 2018.

[33] Q. Sun, S. Meng, and Y. Cui, "Existence results for fractional order differential equation with nonlocal Erdelyi-Kober and generalized Riemann-Liouville type integral boundary conditions at resonance," Advances in Difference Equations, Article ID 243, 16 pages, 2018.

[34] X. Zhang, L. Liu, B. Wiwatanapataphee, and Y. Wu, “The eigenvalue for a class of singular $p$-Laplacian fractional differential equations involving the Riemann-Stieltjes integral boundary condition," Applied Mathematics and Computation, vol. 235, pp. 412-422, 2014.

[35] X. Zhang, L. Liu, Y. Wu, and Y. Cui, "New result on the critical exponent for solution of an ordinary fractional differential problem," Journal of Function Spaces, vol. 2017, Article ID 3976469, 4 pages, 2017.

[36] W. Fan, X. Hao, L. Liu, and Y. Wu, "Nontrivial solutions of singular fourth-order Sturm-Liouville boundary value problems with a sign-changing nonlinear term," Applied Mathematics and Computation, vol. 217, no. 15, pp. 6700-6708, 2011.

[37] L. Liu, B. Liu, and Y. Wu, "Nontrivial solutions for higherorder $m$-point boundary value problem with a sign-changing nonlinear term," Applied Mathematics and Computation, vol. 217, no. 8, pp. 3792-3800, 2010.

[38] K. Zhang, "Nontrivial solutions of fourth-order singular boundary value problems with sign-changing nonlinear terms," Topological Methods in Nonlinear Analysis, vol. 40, no. 1, pp. 5370, 2012.

[39] J. Jiang, J. Henderson, J. Xu, and Z. Fu, "Positive solutions for a system of Neumann boundary value problems of second-order difference equations involving sign-changing nonlinearities," Journal of Function Spaces, vol. 2019, Article ID 3203401, 10 pages, 2019.

[40] A. A. Kilbas, H. M. Srivastava, and J. J. Trujillo, Theory and Applications of Fractional Differential Equations, vol. 204 of North-Holland Mathematics Studies, Elsevier, Amsterdam, The Netherlands, 2006.

[41] I. Podlubny, Fractional Differential Equations, vol. 198 of Mathematics in Science and Engineering, Academic Press, San Diego, Calif, USA, 1999.

[42] S. G. Samko, A. A. Kilbas, and O. I. Marichev, Fractional Integrals and Derivatives, Theory and Applications, Gordon and Breach, Yverdon, Switzerland, 1993.

[43] K. Deimling, Nonlinear Functional Analysis, Springer, Berlin , Germany, 1985.

[44] D. J. Guo and V. Lakshmikantham, Nonlinear Problems in Abstract Cones, vol. 5, Academic Press, Orlando, Fla, USA, 1988. 


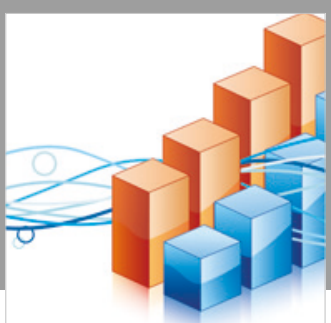

Advances in

Operations Research

\section{-n-m}
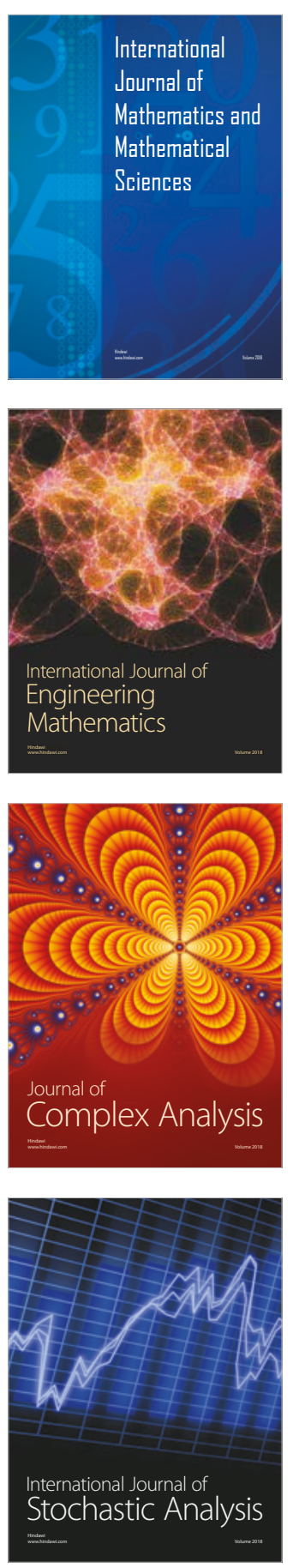
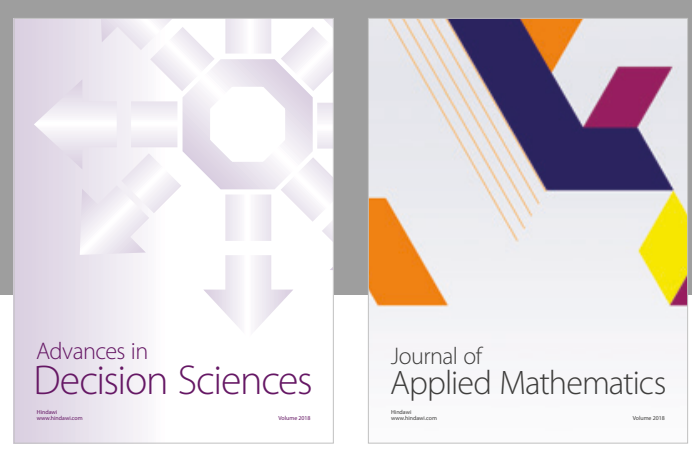

Journal of

Applied Mathematics
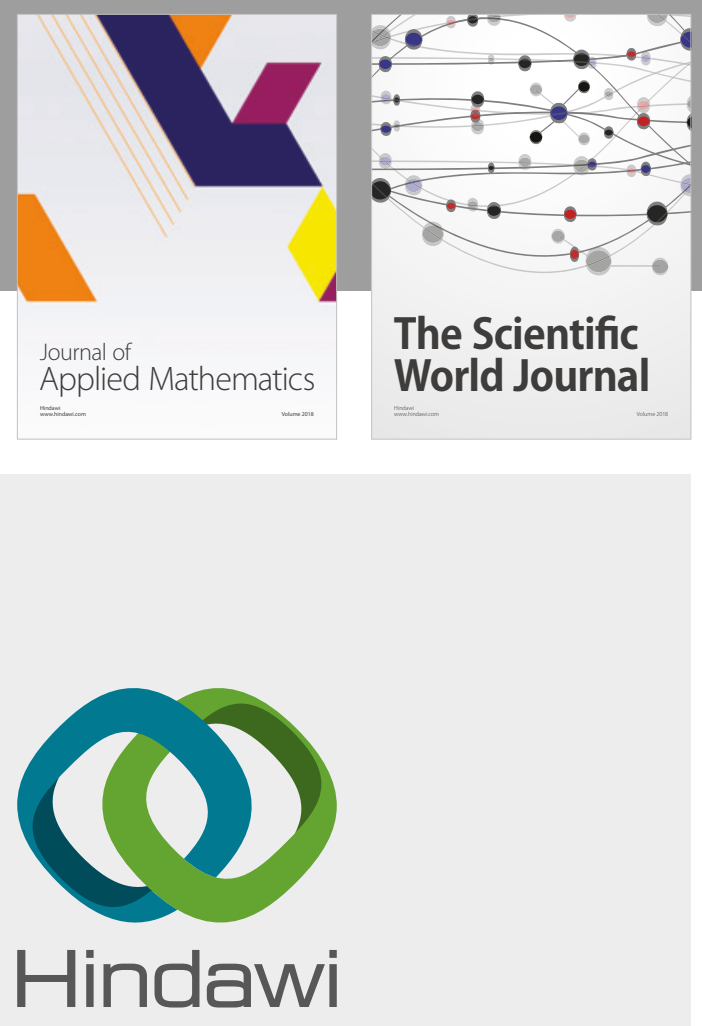

Submit your manuscripts at

www.hindawi.com

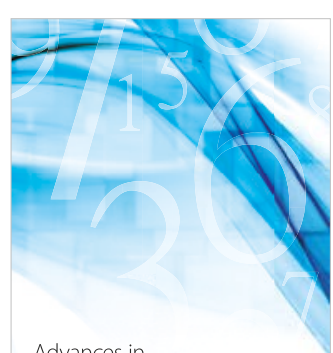

Advances in
Numerical Analysis
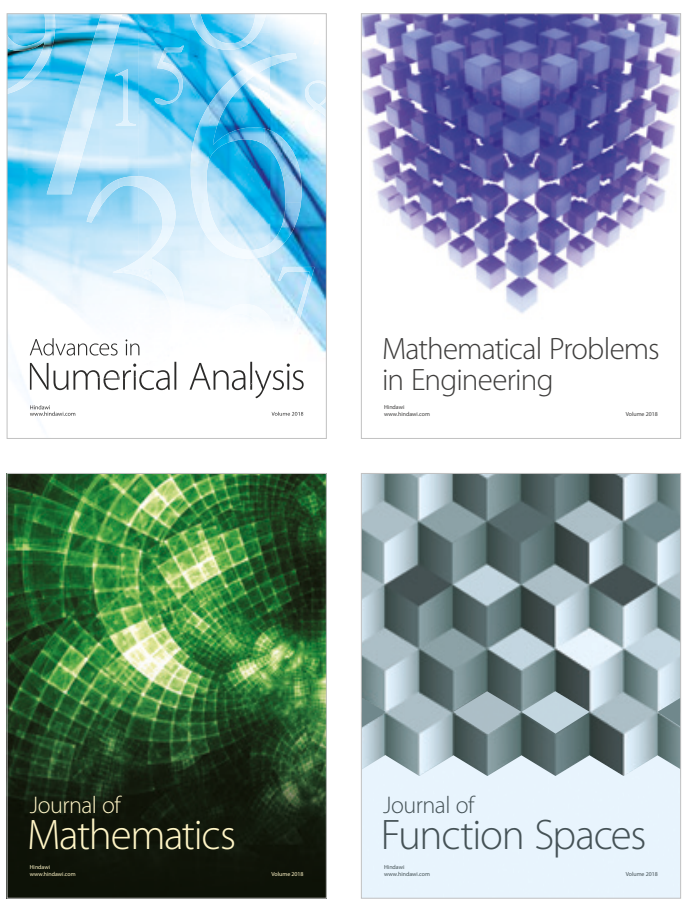

Mathematical Problems in Engineering

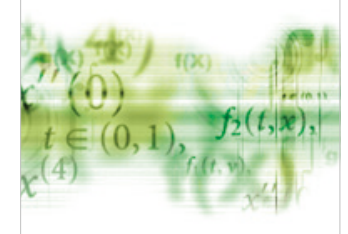

International Journal of

Differential Equations

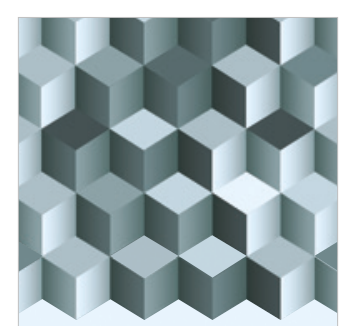

Journal of

Function Spaces

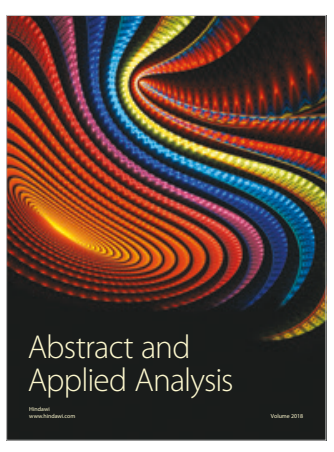

The Scientific

World Journal

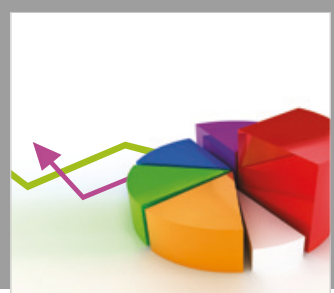

Journal of

Probability and Statistics
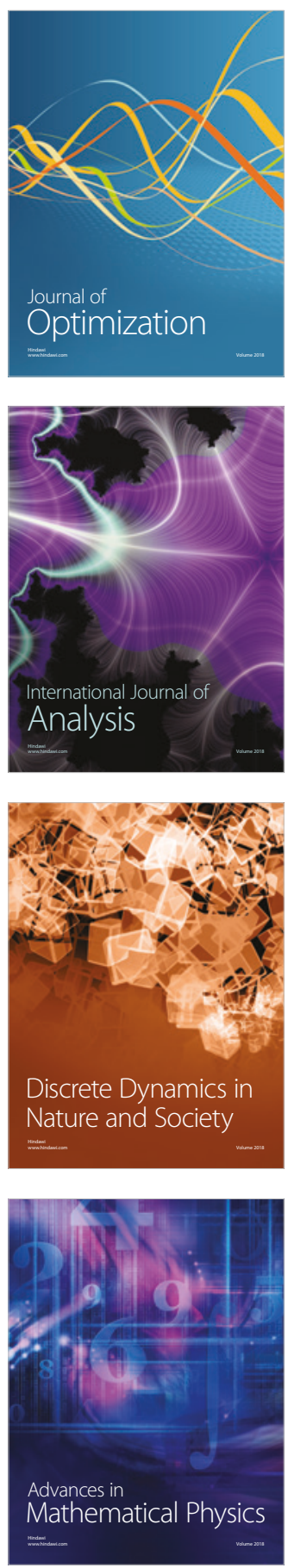